\title{
Local Behavior Analysis for Service Object Based on Intelligent Space
}

\author{
Haitao Li \\ College of Information Science and Engineering \\ Yanshan University \\ Qinhuangdao, HeBei Province, 066004, China \\ lht@ysu.edu.cn
}

\author{
Hanqing Zhang \\ Hebei Institute of Foreign Languages \\ Qinhuangdao, HeBei Province, 066004, China
}

\begin{abstract}
According to the position behavior characteristics of service object, this paper firstly determines the environmental mark point by combining the position point clustering with map topological node. By using the state of family environmental mark point to transfer fitting continuous position trajectory, the overall position and behavior pattern of of service object can be analyzed; the possible target position and residence time, and whether it's in an abnormal state can be determined. At the same time, because of the high computational complexity and poor realtime performance existing in the traditional modeling and identification process, it further introduces the environmental mark point state residence time distribution, combined with the retention time of service object in different markers region, to optimization the model. Finally, this paper detects the service object position, and the experiments show that the model can depict the position movement well, and can help analyze and judge according to the anomaly detection results.
\end{abstract}

Keywords- Position Behavior; Environmental mark point; Anomaly Detection; Trajectory: Service object

\section{INTRODUCTION}

Intelligent home technology aims at establishing a human-centered space with computing and communication ability. By letting the computer to participate in daily activities, users can interact with computer system like other people, and receive humanized services transparently at anytime and anywhere [1-5]. Among them, automatic analysis and recognition of "people's" behavior and intention are of great concern for intelligent home and public places monitoring and other areas. The goal is to get rid of the traditional way of human-computer interaction, let the computer system has the ability to get target behavior information automatically, and make corresponding judgment through analysis, thus making it more intelligent and humanized.

In the intelligent home environment, the service objects are mostly the elderly. Their daily habits have certain rules, and this provides the possibility for us to study and explore the behavior mode[6-8]. Position is one of the most direct information which embodies human activities in the family environment, and the characteristics are easy to extract and represent. For service objects with regular daily activities, they always appear in the similar regions of family space at similar times, and their position behavior models can reflect the behavior rules to a certain extent. Therefore, behavior characteristics can get overall modeling according to position characteristics, and get auxiliary decision and judgment according to action and physiological characteristics.

The study which aims at position behavior habit learning mainly focuses on position regional mapping, position behavior model building and other key issues. $\mathrm{Li}$ proposed a position behavior model of CRF [9], which verifies the activity patterns of the service object. The research directly use the position coordinates as features for representation and training, and experimental data are used for off-line learning and training. The data are huge and of high computational complexity. Document [10] divides the regions of home space, and establishes the HMM model training distribution. The region mainly comes from daily habits and experience, which is lack of self-adaptability of different objects and the environment.

The target behavior analysis based on position trajectory focuses on solving the classification of multidimensional sequence data. Its main purpose is to find or discover abnormal or similar trajectories, while guaranteeing that the classification algorithm can adapt to some minor changes of the trajectory in the space and time scales. Trajectory of service object in family environment is disorderly, and is influenced by time, environment and other factors. Therefore, in the family environment, analysis of continuously moving trajectory of the target position is of little significance. In this paper, we use holographic map node as the initial value of FCM (Fuzzy C-Means, FCM) clustering algorithm[9], get environment mark point collection of family space after stable training , and establish the connection between the real-time position of service object and map environmental mark point. Then, according to the transfer rules of position in the map, directly describe the overall daily habits, or to transfer the position trajectory map into map environmental mark point. At the same time, train daily position behavior model, judge the possible target position and residence time, and judge whether it's in an abnormal condition.

\section{CHOOSING THE ENVIRONMENTAL MARK POINT}

In order to analyze target trajectory in a family environment, we usually need to find some mark points as references for trajectory sequence comparison. The existing literature mostly specify some special positions by artificial method, such as sofa, restaurant, desks and other areas where activities are more frequent. There are more randomness and uncertainty, and can not reflect the position activity rules of different service object. 
To improve the selected mark points, combine the actual distribution of target position point clustering and map node to jointly determine the environmental mark point collection. We set the collection of all tracked target original point in the environment map, indicates the position coordinates, indicates the number of all points collected. Then conduct fuzzy clustering to the position vectors in set. We suppose that it can be divided into classes. FCM machine learning is a classical clustering method, which describe the degree of correspondence between the sample and the classification mainly through the degree of membership, and then obtain the objective function through iterative calculation of new membership and the computing center, which makes the objective function minimum, so as to achieve the classification results. The clustering effect of this algorithm is related to the clustering number and the initial clustering center selection, and is also related to geometric construction of the sample collection and the sorting of the sample. Usually, when there is no available priori information, we choose test method to select different clustering number and initial clustering center to complete the clustering task. In the experiment, the family environment map stores some environment nodes for auxiliary semantic description and dynamic updates of the map, so you pre-set the number and position of nodes in the family map as the initial vector center and number of cluster.

After the training the clustering result tends to be stable, thus proving that residence position of service object with regular activities in home environment is not random. People often stay in some relatively fixed area, and environmental mark points can effectively reflect the distribution, as shown below.

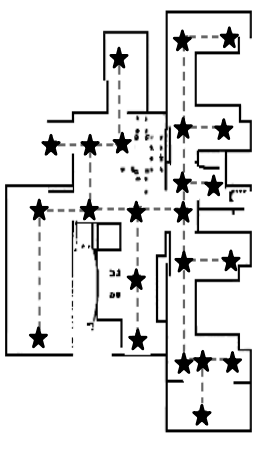

(a)

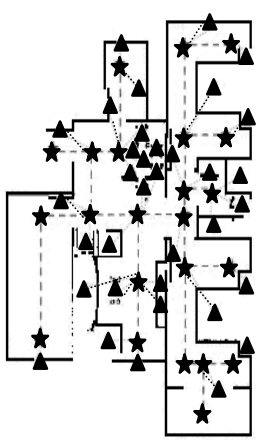

(b) $\star$ Environment topological node

$\Delta$ Position cluster point

(a) Distribution of environment topological node in the map

(b) Distribution of mark point after position clustering in the map

Figure 1. Distribution of environmental mark point

The image above shows the effects of clustering all quantization vectors for the position trajectory on the map. Each cluster center can be regarded as a mark point of the family environment. This method avoids the possible errors of artificial intervention. The result is closer to the actual motion characteristics of the target trajectory, and it improves the robustness of the system.

\section{MODELS OF STATE RESIDENCE}

\section{A. Time distribution of behavior residence time}

After obtaining the point set, we can get position moving state law by calculating the membership degree of the actual trajectory and the symbol point. In practical model training and recognition, often there will be continuous position values observed under the same mark points, namely, service object stays for a period of time in an area. In order to represent the target in different positions and time pattern, we choose a relatively stable position change data, and calculate the frequency and time in different environment mark points. The outputs are shown below.
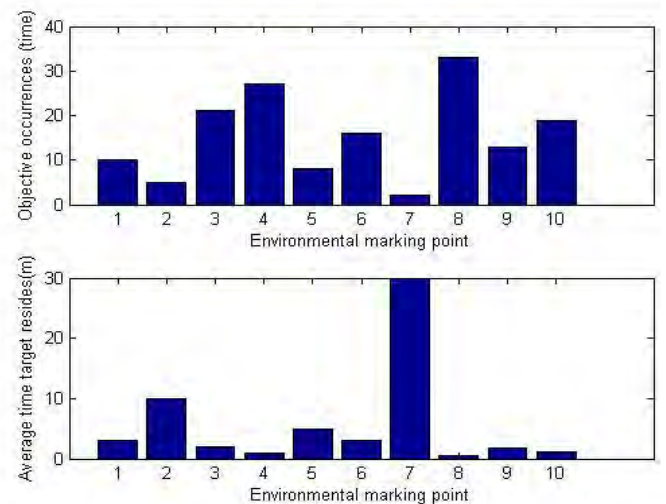

Figure 2.Distribution of behavior residence time

Seen from the results in the image, the frequency and residence time of service objects under different environmental mark point area are not equally distributed. The objects frequently appear in a particular location area, such as the bathroom, sofa, kitchen (No. 3, 4, 8). At the same time the frequency in the bed region (No. 7) is rare, while the time is longer. With decreased activity, people's activity area will be obviously reduced, and tends to be stable, and behavior analysis results are more accurate. In addition, when input data is very messy, since human activities are very frequent, so the activity area would be great, acquired sample data and the dispersion state would be dispersed.

Position moving trajectory of service object can be mapped into the transfer of environmental mark point state. It can be assumed to satisfy the Markov name, and then be modeled in HMM. According to the membership degree of position feature vector to environmental mark point, we establish a traditional HMM to describe the transfer pattern of different points regions, and use the state skip probability to represent a simple state time [8]. But this method doesn't have enough characterization to the state time. For the transferring of position state within the family, different behavior holds up different time. We can ignore such difference in the model training, and we cannot use ordinary uniform distribution or geometric distribution to replace it.

For service objects that are older and have singular activity pattern, long-time position quantization set corresponds to less point state sets. Under this circumstance, computational complexity is high and realtime performance is poor in traditional HMM modeling and recognition process. Therefore, we should consider 
different retention time mark point state, and improve the recognition accuracy and robustness of model.

\section{B. Position behavior model}

A residence time parameter is added into the HMM model, denoted as TSHMM (Time Stay Hidden Markov Model). Each environmental mark point in state transfer sequences corresponds to a random vector, which represents the time distribution of this position. Transition probabilities between environmental states should be considered with the constraints of last state residence time. The relationship between state sequence and time resident are shown below.

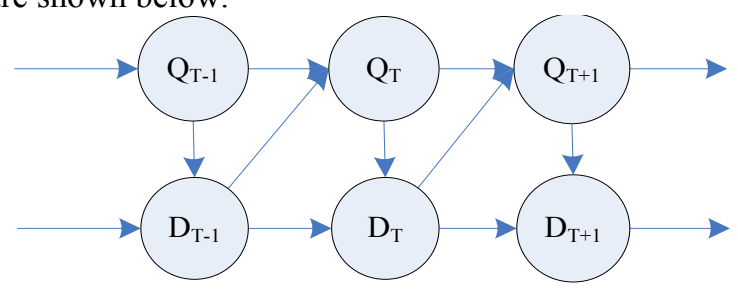

Figure 3.TSHMM Model

We add a pending state to the point set, denoted as $s_{0}$ $S^{\prime}=\left\{s_{0}, s_{1}, \ldots, S_{c}\right\} \quad$ represents the corresponding $c+1$ position state set. Residence time of environment mark point areas is continuous random value. We choose sampling interval $t$ as the basic unit. Set $D=\left\{D_{0}, D_{1}, \ldots, D_{c}\right\} \quad$ respectively represent random vector of residence time in different location area. We believe the residence time in different location areas satisfy the Gauss distribution. Each $D_{i}, i=0,1, \ldots, c$ satisfy Gauss distribution. $\mu_{i}$ represents the time with largest probability among the residence time of each state, and $\mu$ represents the maximum time possible in any state. In this way, $P_{i}(\tau)=P\left(D_{i}=\tau \mid q_{t}=i\right)$ represent the probability that in $t$ moment, residence time in position state $i$ is $\tau$. Among that, $i=0,1, \ldots, c, \tau=1, \ldots, \mu$.

Other two parameters are defined as such methods: one is $\pi_{i}=p\left(q_{1}=s_{i}\right), i=0,1, \ldots, z$, the other is $a_{i j}(\tau)=P\left(q_{t}=j \mid q_{t-1}=i \quad D_{i}=\tau\right), i 、 j=0,1, \ldots, c \quad$, $a_{i j}(\tau)$ represent the probability of transferring from the position $i$ to position $j$, and the residence time at $i$ is $\tau . B=\left\{b_{j}(k)\right\}$ represent the probability of position feature quantization value $v_{k}$ in state $s_{j}$. The probability of service object observation in that state $q_{t} \in S$ can be directly obtained by the coordinate parameters. See equation(1).

$$
q_{t}=s_{i} \mid \arg \min \left(\left|o_{t}-s_{i}\right|\right), \quad s_{i} \in S^{\prime} \quad i=0,1, \ldots, c
$$

Observation sequence $O=\left\{o_{1}, o_{2}, \ldots, o_{m}\right\}$ contains $m$ position feature quantization values. With observation sequence $O$ and model $\lambda$, we can calculate forward variables and backward variables by equation (2) and (4).

$$
\begin{aligned}
& \alpha_{t}(i, \tau)=p\left(o_{1}, o_{2}, \ldots, o_{t}, q_{t}=s_{i}, D_{i}=\tau \mid \lambda\right) \\
& \beta_{t}(i, \tau)=p\left(o_{t+1}, o_{t+2}, \ldots, o_{m}, q_{t}=s_{i}, D_{i}=\tau \mid \lambda\right) \\
& p(O \mid \lambda)=\sum_{i=0}^{c} \sum_{\tau=1}^{\mu} \alpha_{t}(i, \tau) \beta_{t}(i, \tau)
\end{aligned}
$$

By using equation (5) and (6), we can calculate the probability $\xi_{t}(i, j, \tau)$ in state $s_{j}$ at $t+1$, when at $t$, residence time of $s_{i}$ is $\tau$. We can also calculate probability $\gamma_{t}(i, \tau)$ that at ${ }^{t}$, residence time of $s_{i}$ is $\tau$.

$$
\begin{gathered}
\xi_{t}(i, j, \tau)=p\left(q_{t}=s_{i}, D_{i}=\tau, q_{t+1}=s_{j} \mid O, \lambda\right) \\
=\frac{\alpha_{t}(i, \tau) a_{i j}(\tau) b_{j}\left(O_{t+1}\right) \beta_{t+1}(j, 1) p_{j}(1)}{\sum_{i=0}^{c} \sum_{j=0}^{c} \sum_{\tau=1}^{\mu} \alpha_{t}(i, \tau) a_{i j}(\tau) b_{j}\left(O_{t+1}\right) \beta_{t+1}(j, 1) p_{j}(1)}, i \neq j \\
\xi_{t}(i, j, \tau)=\frac{\alpha_{t}(i, \tau) a_{i i}(\tau) b_{i}\left(O_{t+1}\right) \beta_{t+1}(i, \tau+1) p_{i}(\tau+1)}{\sum_{i=0}^{c} \sum_{\tau=1}^{\mu} \alpha_{t}(i, \tau) a_{i i}(\tau) b_{i}\left(O_{t+1}\right) \beta_{t+1}(i, \tau+1) p_{i}(\tau+1)} \\
, i=j \quad \gamma_{t}(i, \tau)=p\left(q_{t}=s_{i}, D_{i}(t)=\tau \mid O, \lambda\right) \\
=\frac{\alpha_{t}(i, \tau) \beta_{t}(i, \tau)}{\sum_{i=0}^{c} \sum_{\tau=1}^{\mu} \alpha_{t}(i, \tau) \beta_{t}(i, \tau)}
\end{gathered}
$$

By traversing $t$ to calculate $\xi_{t}(i, j, \tau)$ and $\gamma_{t}(i, \tau)$, we improve the parameter re-estimation formula. See equation (8)-(10).

$$
\bar{\pi}_{i}=r_{1}(i, 1)
$$

$\overline{a_{i j}(\tau)}=\frac{\text { The number of expectation from } s_{i} \text { to } s_{j}}{\text { The number of expectation from } s_{i}}$

$$
=\frac{\sum_{t=0}^{C-1} \xi_{t}(i, j, \tau)}{\sum_{t=0}^{C-1} \gamma_{t}(i, \tau)}
$$

$$
\begin{aligned}
\overline{b_{j}(k)} & =\frac{\text { the expectaion of } s_{j} \text { for obsered value } s_{i}}{\text { the number expectaion of } s_{j}} \\
& =\frac{\sum_{\tau=1}^{\mu} \sum_{t=0}^{C} \gamma_{t}(j, \tau)}{\sum_{\tau=1}^{\mu} \sum_{t=0}^{C} \gamma_{t}(j, \tau)}
\end{aligned}
$$

Modeling daily habits by using two Markov chain of position state transfer and time state resides to establish TSHMM model, can accurately reflect the state transition pattern of the region. On the one hand, the model will transfer time state into position, and construct model and 
learn it on the whole, on the other hand, it is different from stratified behaviors of general HHMM model. The location and time here in the hierarchical structure is not separated strictly, and time resident is discrete distribution which belongs to the location area state.

\section{EXPERIMENT}

\section{A. Overall behavior analysis experiment}

In the family environment,some regular position trajectory of the service object can be fitted to the environment sign state change. Calculate by overall position behavior model, we can determine the probability of normal and abnormal overall position behavior in a certain period of time, and then according to the transfer pattern of the service object state, we can predict its next position. For example, if the environment state transition of a service object in a certain period of time is detected to be 5-9-7-2-6 (number representations of environmental mark point number, can be manually added to the kitchen, dining room, balcony, semantic description), calculate to determine whether the target is normal, and get that there is maximum probability for the service object to reach No. 10 area.

Distribution and state transfer condition of environmental mark points after fitting position trajectory is shown in Fig 4.

As can be seen, comparing to position moving trajectory, the environment mark point transfer has the advantages of simple structure, easy representation and modeling, and retains the original activity pattern of service object.

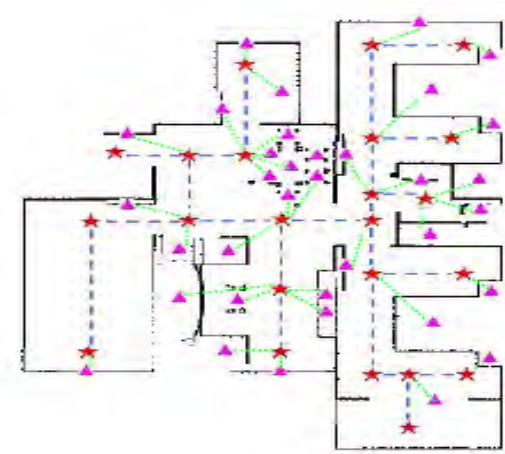

Figure 4. Environmental mark point transfer

Define abbreviations and acronyms the first time they are used in the text, even after they have been defined in the abstract. Abbreviations such as IEEE, SI, MKS, CGS, sc, dc, and rms do not have to be defined. Do not use abbreviations in the title or heads unless they are unavoidable.

\section{B. Abnormal behavior judgment}

For moving trajectory record under normal circumstances, the service object location behavior is normal, and abnormal behavior happens occasionally. To verify the applicability of the model, it needs to do some processing to the data of object position. Artificially add some abnormal position, which make it deviate from normal pattern for the abnormal state detection. Use the normal test sample and artificially intervened normal test samples for experiment, and the results are shown in the table

Table 1 Position Abnormal Behavior Test

\begin{tabular}{|c|c|c|c|c|c|}
\hline \multirow{2}{*}{ Parameter } & \multicolumn{2}{|c|}{$\begin{array}{c}\text { Test Position Sequence Nu } \\
\text { mber }\end{array}$} & \multirow{2}{*}{$\begin{array}{l}\text { Correct rate of Anomaly } \\
\text { Detection }(\%)\end{array}$} & \multirow{2}{*}{$\begin{array}{l}\text { Omission rate } \\
\qquad(\%)\end{array}$} & \multirow{2}{*}{$\begin{array}{l}\text { False Alarm Rate } \\
\qquad(\%)(\%)\end{array}$} \\
\hline & Normal & Abnormal & & & \\
\hline 20 & 85 & 20 & 87.3 & 21 & 35.6 \\
\hline 30 & 67 & 10 & 84.6 & 24 & 23.7 \\
\hline 40 & 34 & 41 & 94.1 & 18.4 & 2.1 \\
\hline
\end{tabular}

It can be seen that even when the state number $c$ is 20 , the system can also maintain the correct rate of more than $85 \%$. The main cause for that when $c$ is 30 , correct rate decrease is that abnormal samples have smaller ratio in the total test samples. The experiments show that TSHMM based on state duration can better reflect behavior pattern of target position, and overall anomaly detection can achieve the desired effect.

\section{CONCLUSION}

Location is one of the most direct information which reflects people's activities at home. By using the state of family environment mark point to transfer fitting continuous position trajectory, the overall position and behavior pattern of of service object can be analyzed; the possible target position and residence time, and whether it's in an abnormal state can be determined. Experiments show that the model can depict the position movement well, and can also complete auxiliary analysis and 
judgment according to the anomaly detection results. Future research is based on the position behavior and integrated with other behaviors, to further analysis the behavior rule of the service object.

\section{ACKNOWLEDGEMENT}

In addition, this work is partially supported by Natural Science Research Project from Hebei Education Department (Grant No. F2012203170 and F2012203188).

\section{REFERENCE}

[1] M.G.Ernesto and G.J.L.Lazaro, Proceedings of. Coveragemapping Method based on a Hardware Model for Mobile-robot Positioning in Intelligent Spaces, IEEE Transactions on Instrumentation and Measurement, vol. 59, pp. 266-282, February 2008.

[2] J.H.Lee, Hashi moto.Controlling Mobile Robots in Distributed Intelligent Sensor Network, IEEE Transactions on Industrial Electronics, vol. 50, pp. 890-902, May 2003.

[3] J. Pineau and M. Montemerlo, Towards Robotic Assistants in Nursing Homes: Challenges and Results, Robotics and Autonomous Systems, vol. 42, pp. 271-281, April 2003.

[4] W.Chung and C.Rhee, Door-Opening Control of a Service Robot Using the Multifingered Robot Hand, IEEE Transactions on
Industrial Electronics, vol. 56, pp. 3975-3984, October 2009.

[5] R. D. Schraft and C. Schaeffer, The Concept of A System for Assisting Elderly or Disabled Persons in Home Environments, Proceedings of the IEEE 24th Annual Conference, vol. 4, pp. 24762481, 1998

[6] S. Thrun, Towards a Framework for Human-robot Interaction, Human Computer Interaction, vol. 19, pp. 9-24, 2004.

[7] M.Hans and W.Baum, Concept of a Hybrid Architecture for Care-O-bot, Proceedings of ROMAN, pp. 407-411, 2001.

[8] STEFANOV D H, BIEN Z,The smart house for older persons and persons with physical disabilities: structure, technology, arrangements, and perspectives, IEEE Trans on Neural System and Rehabilitation Engineering, vol. 2, pp. 228-250, 2004.

[9] Stanford V, Using pervasive computing to deliver elder care, IEEE Pervasive Computing Mobile and Ubiquitous Systems, vol. 1, pp. 10-13, January 2002.

[10] Coen M H and Warshawsky N, Meeting the computational needs of intelligent environments: the metaglue system, Proceedings of the 1st International Workshop on Managing Interactions in Smart Environments. Berlin: Springer-Verlag, pp. 201-212, 1999. 